\title{
A NOTE ON ATTRACTORS FOR COMPACT SETS ${ }^{1}$
}

\author{
J. L. SOLOMON
}

\begin{abstract}
Let $G$ be a closed convex subset of a Banach space $X, f: G \rightarrow G$ a continuous map and $M \subset G$ an attractor for compact sets under $f$. It is shown that if $M$ is not connected, then $M$ has a unique invariant component which is an attractor for points; moreover, for each $x$ in $G$, the set of subsequential limit points of $x$ under $f$ is a subset of this unique invariant component.
\end{abstract}

The following is a long-standing conjecture in fixed point theory: Let $G$ be a closed and bounded convex set in a Banach space and let $f: G \rightarrow G$ be a continuous map. Assume there exists an integer $N>1$ such that $f^{N}$ is compact. Then $f$ has a fixed point (?). If this conjecture were true, it would be a generalization of the Schauder Fixed Point Theorem (the case $N=1$ ). In attempting to provide an answer to this conjecture, Roger D. Nussbaum [3] has the following definition and conjecture which he attributes to F. E. Browder.

Definition 1. Let $X$ be a topological space, $f: X \rightarrow X$ a map and $M$ a nonempty subset of $X$. The set $M$ is said to be an attractor for compact sets under $f$ if (1) $M$ is compact and $f(M) \subset M$, and (2) given any compact set $A \subset X$ and any open neighborhood $U$ of $M$, there exists an integer $N=$ $N(A, U)$ such that $f^{n}(A) \subset U$ for all $n \geqslant N$. If $A=\{x\}$ then $M$ is said to be an attractor for points.

Conjecture 1. Let $G$ be a closed convex subset of a Banach space $X$ and $f$ : $G \rightarrow G$ a continuous map. Assume there exists a set $M \subset G$ which is an attractor for compact sets under $f$. Then $f$ has a fixed point (?).

An affirmative answer to Conjecture 1 would imply an affirmative answer to the mentioned longstanding conjecture since the closure of $f^{N}(G)$ would be an attractor for compact sets under $f$.

If one attempts to provide a negative answer to Conjecture 1, more information as to the class of continuous maps which have attractors for compact sets and/or some knowledge of the topological structure of attractors is desirable. It is in this vein that this note is written-several results on the structure of attractors for compact sets (in the setting of Conjecture 1) are given.

Received by the editors October 20, 1976 and, in revised form, January 19, 1977.

AMS (MOS) subject classifications (1970). Primary 47H99; Secondary 47H10, 54H25.

Key words and phrases. Banach space, attractor for compact sets, component, connected.

1 This paper is based on a portion of the author's dissertation, Some results in nonlinear fixed points theory, Texas A \& M University, 1972.

(1) American Mathematical Society 1977 
THeOREM 1. Let $G$ be a closed convex subset of a Banach space $X$ and $f$ : $G \rightarrow G$ a continuous map. If $M \subset G$ is an attractor for compact sets under $f$, then there exists a unique invariant component of $M$.

Proof. If $M$ is connected the theorem is clearly true. Let us assume that $M$ is not connected and let $\mathcal{C}$ be the collection of all components of $M$. We first show that there exists at most one invariant component of $M$. Suppose that $C_{0}$ and $C_{1}$ are distinct invariant components of $M$. Since $C_{0}$ and $C_{1}$ are disjoint, there exist subsets $D$ and $E$ of $M$ such that $M=D \cup E$ and $D \cap E=\varnothing$ with $C_{0} \subset D$ and $C_{1} \subset E$. Define $\varepsilon=d(E, D)$. Then $\varepsilon>0$ since $D$ and $E$ are compact in $G$. Let $S=\cup_{x \in D} S(x, \varepsilon / 3)$ and $T=$ $\cup_{y \in E} S(y, \varepsilon / 3)$; then $d(S, T) \geqslant \varepsilon / 3$. Pick $z \in C_{0}, w \in C_{1}$ and consider $A=\operatorname{co}(\{z, w\})$. Since $A$ is compact and $S \cup T$ is an open neighborhood of $M$, there exists a positive integer $N=N(A, S \cup T)$ such that $f^{n}(A) \subset S \cup$ $T$ for $n \geqslant N$. Now $C_{0}$ and $C_{1}$ are each invariant and

$$
f^{n}(A)=\left[S \cap f^{n}(A)\right] \cup\left[T \cap f^{n}(A)\right] ;
$$

thus, we have a contradiction to $f^{n}(A)$ being connected since $S \cap T=\varnothing$, $S \cap f^{n}(A) \neq \varnothing$ and $T \cap f^{n}(A) \neq \varnothing$. Hence, the invariant component, if it exists, of $M$ is unique. We now establish the existence of an invariant component of $M$. Let $C$ be an arbitrary but fixed element of $\mathcal{C}$ and consider the sequence $\left\{f^{n}(C)\right\}$. First, suppose that $\left\{f^{n}(C)\right\}$ is contained in a finite number, $k$, of distinct components with $f^{(k+1)}(C) \subset C$. Now choose $D$ and $E$ as above with $C \subset D$ and $f(C) \subset C_{2} \subset E, C_{2} \in \mathcal{C}$. Then $\varepsilon=d(D, E)>0$ and define $S$ and $T$ as before. Pick $x_{0}$ in $C$ and $y_{0}$ in $f(C)$ and let $B=\operatorname{co}\left(\left\{x_{0}, y_{0}\right\}\right)$. There exists a positive integer $N=N(B, S \cup T)$ such that $n \geqslant N$ implies $f^{n}(B) \subset S \cup T$; however, $f^{(k+1) m}(B),(k+1) m \geqslant N, m$ a positive integer, has nonempty intersections with both $S$ and $T$, contradicting $f^{n}(B)$ being connected. Thus, $\left\{f^{n}(C)\right\}$ must lie in an infinite number of distinct components. Let $x_{0}$ be an arbitrary but fixed element of $C$, then the sequence $\left\{f^{n}\left(x_{0}\right)\right\}$ contains a convergent subsequence $\left\{f^{n^{n}}\left(x_{0}\right)\right\}$ converging to $y$ in $M$. Let $y \in C_{y} \in \mathcal{C}$. The subsequence $\left\{f^{n_{i}+1}\left(x_{0}\right)\right\}$ converges to $z=f(y)$ with $z \in C_{z} \in \mathcal{C}$. The proof is complete if $C_{z}=C_{y}$, so assume the contrary. Proceeding as in the first part of this proof, obtain $\varepsilon, D, E, S$ and $T$ with $C_{y} \subset D$ and $C_{z} \subset E$. Since $\left\{f^{n_{1}}\left(x_{0}\right)\right\}$ converges to $y$ and $\left\{f^{n_{i}+1}\left(x_{0}\right)\right\}$ converges to $z$, choose a positive integer $K$ such that if $k \geqslant K, f^{n_{k}}\left(x_{0}\right) \in S(y, \varepsilon / 3)$ and $f^{n_{k}+1}\left(x_{0}\right) \in S(z, \varepsilon / 3)$. Fix $k \geqslant K$ and let $s=f^{n_{k}}\left(x_{0}\right) \in f^{n_{k}}(C), t=$ $f^{n_{k}+1}\left(x_{0}\right) \in f^{n_{k}+1}(C)$, and $A=\operatorname{co}(\{s, t\})$. Since $M$ is an attractor for compact sets, there exists a positive integer $N=N(A, S \cup T)$ such that $f^{n}(A) \subset S \cup$ $T$ for $n \geqslant N$. In particular, for $n=n_{j}-n_{k}$ with $j \geqslant k$ and $n \geqslant N, f^{n}(A) \cap S$ $\neq \varnothing$ and $f^{n}(A) \cap T \neq \varnothing$, which contradicts the connectedness of $f^{n}(A)$. Hence $C_{y}=C_{z}$ and we see that $f\left(C_{y}\right) \subset C_{y}$.

COROLlARY 1. Under the hypothesis of Theorem 1 and the further assumption that $M$ is countable, $f$ has a fixed point. 
Let $x \in X$; the set of subsequential limit points of $x$ under $f$, denoted $\mathcal{L}(x)$, is the set of elements $y$ in $X$ such that there exists a subsequence of $\left\{f^{n}(x)\right\}$ which converges to $y$.

LEMMA 1. Under the hypothesis of Theorem $1, \mathcal{L}(x) \neq \varnothing$ for any $x$ in $G$. Furthermore, if $C_{0}$ is the unique invariant component of $M$, then $\mathcal{L}(x) \subset C_{0}$ for each $x$ in $G$.

Proof. Fix $x_{0}$ in $G$ and consider $\left\{f^{n}\left(x_{0}\right)\right\}$. For $m=1$, there exists a positive integer $N(1)$ such that $f^{N(1)}\left(x_{0}\right) \in U_{1}=\cup_{x \in M} S(x, 1)$. Pick $x_{1}$ in $M$ such that $d\left(x_{1}, f^{N(1)}\left(x_{0}\right)\right)=d\left(f^{N(1)}\left(x_{0}\right), M\right)$. By induction, for each positive integer $m$, there exists a positive integer $N(m)>N(m-1)>\cdots>N(1)$ such that $f^{N(m)}\left(x_{0}\right) \in U_{m}=\cup_{x \in M} S(x, 1 / m)$ and $x_{m}$ contained in $M$ such that $d\left(f^{N(m)}\left(x_{0}\right), x_{m}\right)=d\left(f^{N(m)}\left(x_{0}\right), M\right)$. In this manner we obtain a sequence $\left\{x_{m}\right\} \subset M$ such that $d\left(f^{N(m)}\left(x_{0}\right), x_{m}\right) \rightarrow 0$. Compactness of $M$ implies that there exists a subsequence $\left\{x_{m_{m}}\right\}$ of $\left\{x_{m}\right\}$ which converges to $z \in M$. Clearly $f^{N\left(m_{i}\right)}\left(x_{0}\right) \rightarrow z$ and $\mathcal{L}\left(x_{0}\right) \neq \varnothing$. Let $y$ be an arbitrary element of $\mathcal{L}\left(x_{0}\right)$ and assume $y \in C_{1}$, a component of $M$ distinct from $C_{0}$. Define $A=\operatorname{co}(\{y, w\}), w$ an arbitrary but fixed element of $C_{0}$. Proceeding as in the proof of Theorem 1, we contradict the connectedness of $f^{n}(A)$.

If $X$ is a compact space and $f: X \rightarrow X$ is a continuous mapping, then the closure of the orbit of $x, \operatorname{cl}(O(x))$, is compact. To facilitate the proof of Theorem 2, we include a similar result for mappings in a Banach space which have attractors for compact sets. For some results concerning fix points and $\mathcal{L}(x)$ when $\operatorname{cl}(O(x))$ is compact, see [1], [2].

LEMMA 2. Let $G$ be a closed convex subset of a Banach space $X$ and $f$ : $G \rightarrow G$ a continuous map. If $M \subset G$ is an attractor for compact sets under $f$, then for each $x$ in $G$, the closure of the orbit of $x$ is compact.

Proof. Let $x \in G$ and $\left\{O_{\alpha}\right\}$ be any open covering of $\operatorname{cl}(O(x))$. Let $C_{0}$ be the unique invariant component of $M$. By Lemma $1, \operatorname{cl}(O(x)) \cap C_{0}$ is a nonempty closed subset of $C_{0}$ and therefore compact; thus, there exists $\left\{O_{1}, O_{2}, \ldots, O_{N}\right\} \subset\left\{O_{\alpha}\right\}$ which covers $\operatorname{cl}(O(x)) \cap C_{0}$. We complete the proof by showing there are only a finite number of iterates of $x$ not in $\cup_{i=1}^{N} O_{i}$. Assume there exists $\left\{n_{i}\right\}_{i=1}^{\infty}$, an infinite subset of the positive integers, such that $f^{n_{i}}(x) \notin \cup_{k=1}^{N} O_{k}$ for any $i$. Let $\varepsilon>0$, there exists a positive integer $N_{\varepsilon}$ such that $f^{n}(x) \in \cup_{y \in M} S(y, \varepsilon)$ for $n \geqslant N_{\varepsilon}$ since $M$ is an attractor for compact sets under $f$. Choose smallest value $i_{1}$ such that $f^{n_{1}}(x) \in \cup_{y \in M} S(y, \varepsilon)$ and $y_{1} \in M$ such that $d\left(f^{n_{i_{1}}}(x), y_{1}\right)<\varepsilon$. Having chosen $n_{i_{1}}<n_{i_{2}}<\cdots<n_{i_{k-1}}$ and $y_{1}, y_{2}, \ldots, y_{k-1}$, we choose $n_{k}>n_{i_{k-1}}$ and smallest positive integer $i_{k}$ such that $f^{n_{i k}}(x) \in \cup_{y \in M} S(y, \varepsilon / k)$ and $y_{k} \in M$ such that $d\left(f^{n_{i k}}(x), y_{k}\right)<\varepsilon / k$. Hence, we obtain sequences $\left\{f^{n_{i_{k}}}(x)\right\}$ and $\left\{y_{k}\right\}$. The compactness of $M$ yields the existence of a convergent subsequence-for notational convenience we will not distinguish the subsequence from the sequence; i.e., $y_{k} \rightarrow y_{0}$ in $C_{0}$ and thus $f^{n_{i k}}(x) \rightarrow y_{0}$. Since 
$y_{0} \in \mathcal{L}(x), y_{0} \in \operatorname{cl}(O(x)) \cap C_{0}$ and thus $y_{0}$ is contained in $O_{i}$ for some $i$, $1 \leqslant i \leqslant N$, which yields the contradiction $f^{n_{i k}}(x) \in O_{i}$ for infinitely many values of $k$.

Although this author has been unable to show that the unique invariant component of an attractor (in a Banach space) for compact sets is an attractor for compact sets (except in the case $M$ has only a finite number of components, or by placing some restrictions on $f$ ), we do have the following result.

THEOREM 2. If $C_{0}$ is the unique invariant component of an attractor for compact sets $M$ under a continuous map $f: G \rightarrow G, G$ a closed convex subset of a Banach space, then $C_{0}$ is an attractor for points.

Proof. Without loss of generality, we may assume that an open neighborhood of $C_{0}$ is of the form $U=\cup_{y \in C_{0}} S(y, \varepsilon), \varepsilon>0$. Consider the open covering $\left\{S\left(f^{n}(x), \varepsilon / 2\right): n \geqslant 0\right\}$ of $\operatorname{cl}(O(x))$. From the compactness of $\operatorname{cl}(O(x))$ we obtain a finite subcovering $S\left(f^{n_{1}}(x), \varepsilon / 2\right), \ldots, S\left(f^{n_{k}}(x), \varepsilon / 2\right)$. If $S\left(f^{n_{i}}(x), \varepsilon / 2\right) \cap \operatorname{cl}(O(x))$ is infinite for some $i, 1 \leqslant i \leqslant k$, then $S\left(f^{n_{i}}(x), \varepsilon / 2\right) \cap \mathcal{L}(x) \neq \varnothing$, which implies $S\left(f^{n_{i}}(x), \varepsilon / 2\right) \subset U$. Hence, let $N=\max \left\{m: f^{m}(x) \in S\left(f^{n_{i}}(x), \varepsilon / 2\right)\right.$ where $\left.S\left(f^{n_{i}}(x), \varepsilon / 2\right) \cap \mathcal{L}(x)=\varnothing\right\}$; then it is clear that for $n \geqslant N+1, f^{n}(x) \in U$ and $C_{0}$ is an attractor for points under $f$.

This author feels that if the unique invariant component of an attractor for compact sets is not itself an attractor for compact sets, i.e., one cannot assume without loss in generality that attractors for compact sets are connected, then a counterexample may serve as a counterexample to Conjecture 1.

REMARK. I wish to thank the referee for kindly informing me that a result similar to Lemma 2 was announced in early 1976 by Jack K. Hale at the Conference on Ordinary and Partial Differential Equations, held at the University of Dundee, Scotland, March 30-April 2, 1976, and will appear in the Conference Proceedings.

\section{REFERENCES}

1. L. F. Guseman, Jr. and J. L. Solomon, Subsequential limit points of successive approximations, Proc. Amer. Math. Soc. 34 (1972), 573-577.

2. F. T. Metcalf and T. D. Rogers, The cluster set of sequences of successive approximations, J. Math. Anal. Appl. 31 (1970), 206-212.

3. Roger D. Nussbaum, Some asymptotic fixed point theorems, Trans. Amer. Math. Soc. 171 (1972), 349-375.

Department of Mathematics, Mississippi State University, MissisSipPi State, Mississippi 39762 